\title{
Poly-logarithmic Approximation Algorithms for Directed Vehicle Routing Problems
}

\author{
Viswanath Nagarajan and R. Ravi ${ }^{\star}$ \\ Tepper School of Business, Carnegie Mellon University, Pittsburgh PA 15213. \\ $\{$ viswa, ravi\}@cmu.edu
}

\begin{abstract}
This paper studies vehicle routing problems on asymmetric metrics. Our starting point is the directed $k$-TSP problem: given an asymmetric metric $(V, d)$, a root $r \in V$ and a target $k \leq|V|$, compute the minimum length tour that contains $r$ and at least $k$ other vertices. We present a polynomial time $O\left(\log ^{2} n \cdot \log k\right)$-approximation algorithm for this problem. We use this algorithm for directed $k$-TSP to obtain an $O\left(\log ^{2} n\right)$-approximation algorithm for the directed orienteering problem. This answers positively, the question of poly-logarithmic approximability of directed orienteering, an open problem from Blum et al. [2]. The previously best known results were quasi-polynomial time algorithms with approximation guarantees of $O\left(\log ^{2} k\right)$ for directed $k$-TSP, and $O(\log n)$ for directed orienteering (Chekuri \& Pal [4]). Using the algorithm for directed orienteering within the framework of Blum et al. [2] and Bansal et al. [1], we also obtain poly-logarithmic approximation algorithms for the directed versions of discounted-reward TSP and the vehicle routing problem with time-windows.
\end{abstract}

\section{Introduction}

Vehicle routing problems (VRPs) form a large set of variants of the basic Traveling Salesman Problem, that are also encountered in practice. Some of the problems in this class are the capacitated VRP [10], the distance constrained VRP [15], the Dial-a-Ride problem [19], and the orienteering problem [9]. Many different objectives are encountered in VRPs: for example, minimizing cost of a tour (capacitated VRP \& the Dial-a-Ride problem), minimizing number of vehicles (distance constrained VRP), and maximizing profit (the orienteering problem).

The Operations Research literature contains several papers dealing with exact or heuristic approaches for VRPs (ex. [18, 20,13, 14, 5]). The techniques used in these papers include dynamic programming, local search, simulated annealing, genetic algorithms, branch and bound, and cutting plane algorithms. There has also been some interesting work in approximation algorithms for VRPs. The undirected orienteering problem involves finding a bounded length path starting

* Authors supported in part by NSF grants CCF-0430751 and ITR grant CCR-0122581 (The ALADDIN project). 
at a fixed vertex that covers the maximum number of vertices; Blum et al. [2] obtained the first constant factor approximation algorithm for this problem, which was improved to a factor of 3 in Bansal et al. [1]. Blum et al. also obtained a constant factor approximation algorithm for discounted-reward TSP, a problem motivated by robot navigation. Bansal et al. [1] used orienteering as a subroutine to also obtain poly-logarithmic approximation algorithms for some generalizations of orienteering, namely deadline TSP and vehicle routing problem with time windows.

Most of the work on VRPs focuses on symmetric metric spaces. In asymmetric metrics, the best known approximation guarantee for even the basic traveling salesman problem is $O(\log n)$ [7]; improving this bound is an important open question. Obtaining good approximation algorithms for the directed orienteering problem was stated as an open problem in Blum et al. [2]. Recently, Chekuri \& Pal [4] obtained a general approximation algorithm for a class of VRPs on asymmetric metrics, that runs in quasi-polynomial time. In particular, their result implies an $O(\log n)$-approximation algorithm for the orienteering problem on directed graphs (in quasi-polynomial time). We are not aware of any non-trivial polynomial-time approximation algorithms for this problem. In this paper, we study polynomial time approximation algorithms for some vehicle routing problems on asymmetric metrics.

\subsection{Problem Definition}

All the problems that we consider are defined over an asymmetric metric space $(V, d)$ on $|V|=n$ vertices. In the directed $k$-TSP problem, we are given a root $r \in V \&$ a target $k \leq n$, and the goal is to compute a minimum length tour that contains $r$ and at least $k$ other vertices. Any tour containing the root $r$ is called an $r$-tour. Directed $k$-TSP is a generalization of the asymmetric traveling salesman problem (ATSP). A related problem is the minimum ratio ATSP problem, which involves finding a tour containing the root $r$ that minimizes the ratio of the length of the tour to the number of vertices in it. If the requirement that the tour contain the root is dropped, the ratio problem becomes the minimum mean weight cycle problem, which is solvable in polynomial time. However, the rooted version which we are interested in is NP-complete.

In the orienteering problem, we are given a metric space, a specified origin $s$ and a length bound $D$, and the goal is to find a path of length at most $D$, that starts at $s$ and visits the maximum number of vertices. We actually consider a more general version of this problem, which is the directed version of point-to-point orienteering [1]. In the directed orienteering problem, we are given specified origin $s \&$ destination $t$ vertices, and a length bound $D$, and the goal is to compute a path from $s$ to $t$ of length at most $D$, that visits the maximum number of vertices. The orienteering problem can also be extended to the setting where there is some profit at each vertex, and the goal is to maximize total profit.

Many problems we deal with in this paper have the following form, where $\mathcal{S}$ is a feasible set, $C: \mathcal{S} \rightarrow \mathbb{R}^{+}$is a cost function, $N: \mathcal{S} \rightarrow \mathbb{N}$ is a coverage 
function, and $k$ is the target:

$$
\min \{C(x): x \in \mathcal{S}, N(x) \geq k\}
$$

For example, in the $k$-TSP problem, $\mathcal{S}$ is the set of all tours containing $r$, and for any $x \in \mathcal{S}, C(x)$ is the length of tour $x$ and $N(x)$ is the number of vertices (other than $r$ ) covered in the tour. For any problem of the above form, a polynomial time algorithm $\mathcal{A}$ is said to be an $(\alpha, \beta)$ bi-criteria approximation if on each problem instance, $\mathcal{A}$ obtains a solution $y \in \mathcal{S}$ satisfying $C(y) \leq \alpha \cdot O P T$ and $N(y) \geq \frac{k}{\beta}$, where $O P T=\min \{C(x): x \in \mathcal{S}, N(x) \geq k\}$ is the optimal value of this instance.

\subsection{Results \& Paper Outline}

We present a polynomial time $O\left(\log ^{2} n \cdot \log k\right)$-approximation algorithm for the directed $k$-TSP problem. This is based on an $O\left(\log ^{2} n\right)$-approximation algorithm for the minimum ratio ATSP problem. To the best of our knowledge, this problem has not been studied earlier. An important ingredient in this algorithm is a splitting-off theorem on directed Eulerian graphs due to Frank [6] and Jackson [12]. This algorithm is described in Section 2. Our proof also implies a $\lceil\log n\rceil$ upper bound on the integrality gap of a natural LP relaxation for ATSP.

We then use the approximation algorithm for minimum ratio ATSP, to obtain a bi-criteria approximation algorithm for the directed $k$-path problem (Section 3). We also observe that the reductions in Blum et al. [2] \& Bansal et al. [1] (in undirected metrics) from the $k$-path problem to the orienteering problem, can be easily adapted to the directed case. Together with the approximation algorithm for the directed $k$-path problem, we obtain an $O\left(\log ^{2} n\right)$ approximation guarantee for directed orienteering. This answers in the affirmative, the question of polylogarithmic approximability of directed orienteering [2].

Finally, we note that the techniques used for discounted-reward TSP [2], and vehicle routing with time-windows [1], also work in the directed setting. Since these algorithms use the orienteering (or minimum excess) problem in a black-box fashion, our results imply approximation algorithms with guarantees: $O\left(\log ^{2} n\right)$ for discounted-reward TSP, and $O\left(\log ^{4} n\right)$ for VRP with time-windows.

In independent work, Chekuri et al. [3] also obtain many of the results reported in this paper, although via different techniques. Their approximation guarantees are slightly better than what we obtain for these prblems. They obtain an $O\left(\log ^{3} k\right)$-approximation algorithm for the directed $k$-TSP problem, and an $O\left(\log ^{2} O P T\right)$-approximation algorithm for directed orienteering (where $O P T \leq n$ is the optimal value of the orienteering instance).

\section{The Directed $k$-TSP Problem}

The directed $k$-TSP problem is a generalization of the asymmetric traveling salesman problem (ATSP), for which the best known approximation guarantee 
is $O(\log n)$. In this section, we obtain an $O\left(\log ^{2} n \cdot \log k\right)$-approximation algorithm for this problem. We first obtain an $O\left(\log ^{2} n\right)$-approximation algorithm for minimum ratio ATSP (Theorem 5), and then show how this implies the result for directed $k$-TSP (Theorem 6). Our algorithm for minimum ratio ATSP is based on the integrality gap (Theorem 2) of a suitable LP relaxation for ATSP, which we study next.

\subsection{A linear relaxation for ATSP}

In this section, we consider the following LP relaxation for ATSP, where $(V, d)$ is the input metric.

$$
\begin{aligned}
& \min \sum_{e} d_{e} \cdot z_{e} \\
& \text { s.t. } \\
& (A L P) \\
& \begin{array}{cl}
z\left(\delta^{+}(v)\right)=z\left(\delta^{-}(v)\right) & \forall v \in V \\
z\left(\delta^{+}(S)\right) \geq 1 & \forall \phi \neq S \neq V \\
z_{e} \geq 0 & \forall \operatorname{arcs} \mathrm{e}
\end{array}
\end{aligned}
$$

This relaxation was also studied in Vempala \& Yannakakis [21], where the authors proved a structural property about basic solutions to $(A L P)$. We show that the integrality gap of $(A L P)$ is at most $\lceil\log n\rceil$. The following stronger LP relaxation (with additional degree $=1$ constraints) was shown to have an integrality gap of at most $\lceil\log n\rceil$ in Williamson [22].

$$
\begin{array}{rll} 
& \min \sum_{e} d_{e} \cdot z_{e} & \\
\text { s.t. } & z\left(\delta^{+}(v)\right)=1 & \forall v \in V \\
\left(A L P^{\prime}\right) & z\left(\delta^{-}(v)\right)=1 & \forall v \in V \\
& z\left(\delta^{+}(S)\right) \geq 1 & \forall \phi \neq S \neq V \\
& z_{e} \geq 0 & \forall \operatorname{arcs} \mathrm{e}
\end{array}
$$

However, we are not aware of any previously known upper bound on the integrality gap of $(A L P)$. We first give an independent proof that upper bounds the integrality gap of $(A L P)$ (Theorem 2), and then show that for any asymmetric metric $(V, d)$, the optimal values of $(A L P)$ and $\left(A L P^{\prime}\right)$ coincide (Theorem 3). Our proof makes use of the following directed splitting-off theorem due to Mader [16].

Theorem 1 (Mader [16]) Let $D=(U+x, A)$ be a directed graph such that indegree equal to outdegree at $x$, and the directed connectivity between any pair of vertices in $U$ is at least $k$. Then for every arc $(x, v) \in A$ there exists an arc $(u, x) \in A$ so that after replacing the two arcs $(u, x) \&(x, v)$ by an arc $(u, v)$, the directed connectivity between every pair of vertices in $U$ remains at least $k$.

This operation of replacing two $\operatorname{arcs}(u, x) \&(x, v)$ by the single $\operatorname{arc}(u, v)$ is called splitting-off.

Theorem 2 The integrality gap of $(A L P)$ is at most $\lceil\log n\rceil$. 
Proof: This proof has the same outline as the proof for the stronger LP relaxation $\left(A L P^{\prime}\right)$ in Williamson [22]. We use the $\lceil\log n\rceil$ approximation algorithm for ATSP due to Frieze et al. [7], which works by computing cycle covers repeatedly (in at most $\lceil\log n\rceil$ iterations). In this algorithm, if $U \subseteq V$ is the set of representative vertices in some iteration, the cost incurred in this iteration equals the minimum cycle cover on $U$. Let $A L P(U)$ denote the LP relaxation $A L P$ restricted to a subset $U$ of the original vertices, and $\operatorname{opt}(A L P(U))$ its optimal value. Then we have:

Claim 1 For any subset $U \subseteq V$, the minimum cycle cover on $U$ has cost at most opt $(A L P(U))$.

Proof: Consider the following linear relaxation for cycle cover.

$$
\begin{array}{cc}
\min \sum_{e} d_{e} \cdot x_{e} & \\
\text { s.t. } & \\
x\left(\delta^{+}(v)\right)-x\left(\delta^{-}(v)\right)=0 & \forall v \in U \\
x\left(\delta^{+}(v)\right) \geq 1 & \forall v \in U \\
x_{e} \geq 0 & \forall \operatorname{arcs} \mathrm{e}
\end{array}
$$

These constraints are equivalent to a circulation problem on network $N$ which contains two vertices $v_{\text {in }} \& v_{\text {out }}$ for each vertex $v \in U$. The $\operatorname{arcs}$ in $N$ are: $\left\{\left(u_{\text {out }}, v_{\text {in }}\right): \forall u, v \in U, u \neq v\right\}$, and $\left\{\left(v_{\text {in }}, v_{\text {out }}\right): \forall v \in U\right\}$. The cost of each $\left(u_{\text {out }}, v_{\text {in }}\right)$ arc is $d(u, v)$, and each $\left(v_{\text {in }}, v_{\text {out }}\right)$ arc costs 0 . It is easy to see that the minimum cost circulation on $N$ that places at least one unit of flow on each arc in $\left\{\left(v_{\text {in }}, v_{\text {out }}\right): \forall v \in U\right\}$ is exactly the optimal solution to $(C L P)$. But the linear program for minimum cost circulation is integral (network matrices are totally unimodular, c.f. [17]), and so is $(C L P)$.

Any integral solution to $(C L P)$ defines an Eulerian subgraph $H$ with each vertex in $U$ having degree at least 1. Each connected component $C$ of $H$ is Eulerian and can be shortcut to get a cycle on the vertices of $C$. Since triangle inequality holds, the cost of each such cycle is at most that of the original component. So this gives a cycle cover of $U$ of cost at most opt $(C L P(U))$, the optimal value of $(C L P)$. But the linear program $A L P(U)$ is more constrained than $C L P(U)$; so the minimum cycle cover on $U$ costs at most opt $(A L P(U))$.

We now establish the monotonicity property of $A L P$, namely:

$$
\operatorname{opt}(A L P(U)) \leq \operatorname{opt}(A L P(V)) \quad \forall U \subseteq V
$$

Consider any subset $U \subseteq V$, vertex $v \in U$, and $U^{\prime}=U-v$; we will show that $\operatorname{opt}\left(A L P\left(U^{\prime}\right)\right) \leq \operatorname{opt}(A L P(U))$. Let $z$ be any fractional solution to $A L P(U)$ so that $L \cdot z$ is integral for some large enough $L \in \mathbb{N}$. Define a multigraph $H$ on vertex set $U$ with $L \cdot z_{w_{1}, w_{2}}$ arcs going from $w_{1}$ to $w_{2}$ (for all $w_{1}, w_{2} \in U$ ). From the feasibility of $z$ in $A L P(U), H$ is Eulerian and has arc-connectivity at least $L$. Now applying Theorem 1 repeatedly on vertex $v \in U$ (until its degree is zero), we obtain a multigraph $H^{\prime}$ on $U^{\prime}=U-v$ such that the arc-connectivity of $H^{\prime}$ is still at least $L$. Further, due to the triangle inequality, the total cost of $H^{\prime}$ is at most that of $H$. Finally, scaling down $H^{\prime}$ by $L$ we obtain a fractional solution to 
$A L P\left(U^{\prime}\right)$ of cost at most $d \cdot z$. Thus, opt $\left(A L P\left(U^{\prime}\right)\right) \leq \operatorname{opt}(A L P(U))$, and using this inductively we have monotonicity for $A L P$.

This suffices to prove the theorem, as the cost incurred in each iteration of the Frieze et al. [7] algorithm can be bounded by opt $(A L P(V))$, and there are at most $\lceil\log n\rceil$ iterations.

We note that in order to prove the monotonicity property for the linear program $\left(A L P^{\prime}\right)$, Williamson [22] used the equivalence of $\left(A L P^{\prime}\right)$ and the HeldKarp bound [11], and showed that the Held-Karp lower bound is monotone. Using splitting-off, we obtain a more direct proof of monotonicity. In fact, we can prove a stronger statement than Theorem 2 , which relates the optimal values of $(A L P)$ and $\left(A L P^{\prime}\right)$. It was shown in [22] that the optimal value of $\left(A L P^{\prime}\right)$ equals the Held-Karp lower bound [11]; so the next theorem shows that for any ATSP instance, the values of the Help-Karp bound, $\left(A L P^{\prime}\right)$ and $(A L P)$ are all equal. A similar result for the symmetric case was proved in Goemans \& Bertsimas [8], which was also based on splitting-off (for undirected graphs).

Theorem 3 The optimal values of $(A L P)$ and $\left(A L P^{\prime}\right)$ are equal.

Proof: Clearly the optimal value of $\left(A L P^{\prime}\right)$ is at most that of $(A L P)$. We will show that any fractional solution $z$ to $(A L P)$ can be modified to a fractional solution $z^{\prime}$ to $\left(A L P^{\prime}\right)$, such that $\sum_{e} d_{e} \cdot z_{e}^{\prime} \leq \sum_{e} d_{e} \cdot z_{e}$, which would prove the theorem. As in the proof of Theorem 2 , let $L \in \mathbb{N}$ be large enough so that $L \cdot z$ is integral, and let $H$ denote a multi di-graph with $L \cdot z_{u, v}$ arcs from $u$ to $v$, for all $u, v \in V$. From the feasibility of $z$ in $(A L P)$, we know that $H$ is Eulerian and has arc-connectivity at least $L$.

If some $v \in V$ has degree strictly greater than $L$, we reduce its degree by one as follows. Let $v^{\prime}$ be any vertex in $V \backslash v$, and $\mathcal{P}_{v, v^{\prime}}$ denote a minimal set of arcs that constitutes exactly $L$ arc-disjoint paths from $v$ to $v^{\prime}$. Due to minimality, the number of $\operatorname{arcs}$ in $\mathcal{P}_{v, v^{\prime}}$ incident to $v$ is exactly $L$ and they are all $\operatorname{arcs}$ leaving $v$. Since the degree of $v$ is at least $L+1$, there is an $\operatorname{arc}(v, w) \in H \backslash \mathcal{P}_{v, v^{\prime}}$. Applying Theorem 1 to arc $(v, w)$, we obtain arc $(u, v) \in H \backslash \mathcal{P}_{v, v^{\prime}}$ such that the arc-connectivity of vertices $V \backslash v$ in $H^{\prime}=(H \backslash\{(u, v),(v, w)\}) \cup(u, w)$ remains at least $L$. Further, by the choice of $(v, w), \mathcal{P}_{v, v^{\prime}} \subseteq H^{\prime}$; so the arc-connectivity from $v$ to $v^{\prime}$ in $H^{\prime}$ is at least $L$. Since $H^{\prime}$ is Eulerian, it now follows that the arcconnectivity of vertices $V$ in $H^{\prime}$ is also at least $L$. Thus we obtain a multigraph $H^{\prime}$ from $H$ which maintains connectivity and decreases the degree of vertex $v$ by 1 . Repeating this procedure for all vertices in $V$ having degree greater than $L$, we obtain (an Eulerian) multigraph $G$ having arc-connectivity $L$ such that the degree of each vertex equals $L$.

Note that in the degree reducing procedure above, the only operation we used was splitting-off. Since $d$ satisfies triangle inequality, the total cost of arcs in $G$ (under length $d$ ) is at most that of $H$. Finally, scaling down $G$ by $L$, we obtain the claimed fractional solution $z^{\prime}$ to $\left(A L P^{\prime}\right)$ 


\subsection{Minimum ratio ATSP}

We now describe the $O\left(\log ^{2} n\right)$-approximation algorithm for minimum ratio ATSP, which uses Theorem 2. In addition, we require the following strengthening of Mader's splitting-off Theorem, in the case of Eulerian digraphs.

Theorem 4 (Frank [6] (Theorem 4.3) and Jackson [12]) Let $D=(U+$ $x, A)$ be a directed Eulerian graph. For each arc $f=(x, v) \in A$ there exists an arc $e=(u, x) \in A$ so that after replacing arcs e \& $f$ by arc $(u, v)$, the directed connectivity between every pair of vertices in $U$ is preserved.

Theorem 5 There is a polynomial time $O\left(\log ^{2} n\right)$-approximation algorithm for the minimum ratio ATSP problem.

Proof: The approximation algorithm for minimum ratio ATSP is based on the following LP relaxation for this problem.

$$
\begin{array}{ccl} 
& \min \sum_{e} d_{e} \cdot x_{e} & \\
\text { s.t. } & & \\
x\left(\delta^{+}(v)\right)=x\left(\delta^{-}(v)\right) & \forall v \in V \\
x\left(\delta^{+}(S)\right) \geq y_{v} & \forall S \subseteq V-\{r\} \forall v \in S \\
(R L P) & \sum_{v \neq r} y_{v} \geq 1 & \\
x_{e} \geq 0 & & \forall v \in V-\{r\} \\
0 \leq y_{v} \leq 1 & \operatorname{arcs} \mathrm{e}
\end{array}
$$

To see that this is indeed a relaxation, consider the optimal integral $r$-tour $C^{*}$ that covers $l$ vertices (excluding $r$ ). We construct a solution to $(R L P)$ by setting $y_{v}=\frac{1}{l}$ for all vertices $v \in C^{*}$, and $x_{e}=\frac{1}{l}$ for all $\operatorname{arcs} e \in C^{*}$. It is easy to see that this solution is feasible and has cost $\frac{d\left(C^{*}\right)}{l}$ which is the optimal ratio. The linear program $(R L P)$ can be solved in polynomial time using the Ellipsoid algorithm. The algorithm is as follows:

1. Let $(x, y)$ denote an optimal solution to $(R L P)$.

2. Discard all vertices $v \in V \backslash r$ with $y_{v} \leq \frac{1}{2 n}$; all remaining vertices have $y$-values in the interval $\left[\frac{1}{2 n}, 1\right]$.

3. Define $g=\left\lceil\log _{2} n+1\right\rceil$ groups of vertices where group $G_{i}$ (for $i=1, \cdots, g$ ) consists of all vertices $v$ having $y_{v} \in\left(\frac{1}{2^{i}}, \frac{1}{2^{i-1}}\right]$.

4. Run the Frieze et al. [7] algorithm on each of $G_{i} \cup\{r\}$ and output the $r$-tour with the smallest ratio.

Note that the total $y$-value of vertices remaining after step 2 is at least $1 / 2$. Consider any group $G_{i}$; let $L_{i} \in \mathbb{N}$ be large enough so that $L_{i} \cdot 2^{i} \cdot x$ is integral. Define a multigraph $H_{i}$ with $L_{i} \cdot 2^{i} \cdot x_{u, v}$ arcs from $u$ to $v$ for all $u, v \in V$. Below, for a directed graph $D$ and vertices $u, v \in D$ the directed arc-connectivity from $u$ to $v$ is denoted $\lambda(u, v ; D)$. From the feasibility of $x$ in $R L P$, it is clear that $H_{i}$ is Eulerian. Further, for all $v \in G_{i}, \lambda\left(r, v ; H_{i}\right)=\lambda\left(v, r ; H_{i}\right) \geq L_{i} \cdot 2^{i} \cdot y_{v} \geq L_{i}$. Now we split-off vertices in $V \backslash\left(G_{i} \cup\{r\}\right)$ one by one, using Theorem 4, which preserves the arc-connectivity of $G_{i} \cup\{r\}$. This results in an Eulerian multigraph $H_{i}^{\prime}$ on 
vertices $G_{i} \cup r$ satisfying $\lambda\left(r, v ; H_{i}^{\prime}\right), \lambda\left(v, r ; H_{i}^{\prime}\right) \geq L_{i}$ for all $v \in G_{i}$. Further, due to triangle inequality the total weight of $\operatorname{arcs}$ in $H_{i}^{\prime}$ is at most that in $H_{i}$. Now, scaling down $H_{i}^{\prime}$ by $L_{i}$, we obtain a fractional solution $z^{i}$ to $A L P\left(G_{i} \cup\{r\}\right)$ of $\operatorname{cost} d \cdot z^{i} \leq 2^{i}(d \cdot x)$. Now Theorem 2 implies that there exists an $r$-tour on $G_{i}$ of cost at most $\beta=\lceil\log n\rceil$ times $d \cdot z^{i}$. In fact, the Frieze et al. [7] algorithm applied on $G_{i}+r$ produces such a tour. We now claim that one of the $r$-tours found in step 4 (over all $i=1, \cdots g$ ) has a small ratio:

$$
\min _{i=1}^{g} \frac{\beta\left(d \cdot z^{i}\right)}{\left|G_{i}\right|} \leq \min _{i=1}^{g} \frac{2^{i} \beta(d \cdot x)}{\left|G_{i}\right|} \leq \frac{\beta \sum_{i=1}^{g} d \cdot x}{\sum_{i=1}^{g}\left|G_{i}\right| / 2^{i}} \leq 4 g \beta \cdot(d \cdot x)
$$

The last inequality follows from the fact that after step $2, \frac{1}{2} \leq \sum_{v \neq r} y_{v} \leq$ $\sum_{i=1}^{g} \frac{1}{2^{i-1}}\left|G_{i}\right|=2 \sum_{i=1}^{g} \frac{\left|G_{i}\right|}{2^{i}}$, since there is a total $y$-weight of at least $1 / 2$ even after step 2. Thus we have a $4 g \beta=O\left(\log ^{2} n\right)$ approximation algorithm for minimum ratio ATSP.

We note that for this proof of Theorem 5 to work, just a bound on the integrality gap of $\left(A L P^{\prime}\right)[22]$ is insufficient. The Eulerian multigraph $H_{i}^{\prime}$ that gives rise to the fractional ATSP solution $z^{i}$ on $G_{i} \cup\{r\}$ may not have degree $L_{i}$ at all vertices; so $z^{i}$ may be infeasible for $A L P^{\prime}\left(G_{i} \cup\{r\}\right)$. This is the reason we need to consider the LP relaxation $(A L P)$.

\section{$2.3 \quad$ Directed $k$-TSP}

We now describe how minimum ratio ATSP can be used to obtain an approximation algorithm for the directed $k$-TSP problem.

Theorem 6 There is a polynomial time $O\left(\log ^{2} n \cdot \log k\right)$ approximation algorithm for the directed $k$-TSP problem.

Proof: We use the $\alpha=O\left(\log ^{2} n\right)$-approximation algorithm for the related minimum ratio ATSP problem. Let $O P T$ denote the optimal value of the directed $k$-TSP instance. By performing binary search, we may assume that we know the value of $O P T$ within a factor 2 . We only consider vertices $v \in V$ satisfying $d(r, v), d(v, r) \leq O P T$; this does not affect the optimal solution. Then we invoke the minimum ratio ATSP algorithm repeatedly (each time restricted to the currently uncovered vertices) until the total number of covered vertices $t \geq \frac{k}{2}$. Note that for every instance of the ratio problem that we solve, there is a feasible solution of ratio $\leq \frac{2 \cdot O P T}{k}$ (namely, the optimal $k$-TSP tour covering at least $k / 2$ residual vertices). Thus we obtain an $r$-tour on $t \geq \frac{k}{2}$ vertices having ratio $\leq \frac{2 \alpha \cdot O P T}{k}$; so the length of this $r$-tour is at most $\frac{2 \alpha t \cdot O P T}{k}$. Now, we split this $r$-tour into $l=\left\lceil\frac{2 t}{k}\right\rceil$ di-paths, each containing at least $\frac{t}{l} \geq \frac{k}{4}$ vertices (this can be done in a greedy fashion). By averaging, the minimum length di-path in this collection has length at most $\frac{2 \alpha t O P T / k}{l} \leq \alpha \cdot O P T$. Joining the first and last vertices in this di-path to $r$, we obtain an $r$-tour containing at least $\frac{k}{4}$ vertices, of length at most $(\alpha+2) \cdot O P T$. So we get an $(O(\alpha), 4)$ bi-criteria approximation 
for directed $k$-TSP. This algorithm can now be used as follows: until $k$ vertices are covered, repeat: if $k^{\prime}$ denotes the number of vertices covered so far, run the bi-criteria approximation algorithm with a target of $k-k^{\prime}$, restricted to currently uncovered vertices. A standard set cover based analysis implies that this is an $O(\alpha \cdot \log k)$-approximation algorithm for directed $k$-TSP.

\section{The Directed Orienteering Problem}

In this section, we consider the orienteering problem in asymmetric metrics. As mentioned before, this is in fact the directed counterpart of the point-to-point orienteering problem [1]. We adapt the framework of Blum et al. [2] (for undirected orienteering) to the directed case. The algorithm for directed orienteering is based on the following sequence of reductions: directed $k$-path to minimum ratio ATSP (Theorem 7 ), directed minimum excess to directed $k$-path (Theorem 8), and directed orienteering to directed minimum excess (Theorem 9). The last two reductions are identical to the corresponding reductions for undirected orienteering in Blum et al. [2] and Bansal et al. [1]. The directed $k$-path problem is the following: given an asymmetric metric $(V, d)$, origin $(s) \&$ destination $(t)$ vertices, and a target $k$, find an $s$ - $t$ di-path of minimum length that visits at least $k$ other vertices. We prove the following bi-criteria approximation guarantee for this problem.

Theorem 7 A $\rho$-approximation algorithm for minimum ratio ATSP implies a $(3,4 \rho)$ bi-criteria approximation algorithm for the directed $k$-path problem.

Proof: We assume (by performing a binary search) that we know the optimal value $O P T$ of the directed $k$-path instance within a constant factor, and let $G$ denote the directed graph corresponding to metric $(V, d)$ (which has an arc of length $d(u, v)$ from $u$ to $v$ for every pair of vertices $u, v \in V)$. We modify graph $G$ to obtain graph $H$ as follows: (a) discard all vertices $v$ such that $d(s, v)>O P T$ or $d(v, t)>O P T$; and (b) add an extra arc from $t$ to $s$ of length $O P T$. In the rest of this proof, we refer to the shortest path metric induced by $H$ as $(V, l)$. Note that each tour in metric $l$ corresponds to a tour in graph $H$ (using shortest paths in $H$ for each metric arc); below, any tour in metric $l$ will refer to the corresponding tour in graph $H$. Since there is an $s$ - $t$ path of length $O P T$ (in metric $d$ ) covering $k$ vertices, appending the $(t, s)$ arc, we have an $s$-tour $\sigma^{*}$ of length at most $2 \cdot O P T$ (in metric $l$ ) covering $k+1$ vertices.

Now, we run the minimum ratio ATSP algorithm with root $s$ in metric $l$ repeatedly until either (1) $\frac{k}{2}$ vertices are covered and the extra $(t, s)$ arc is never used in the current tour in graph $H$; or (2) the extra $(t, s)$ arc is used for the first time in the current tour in $H$. Let $\sigma$ be the $s$-tour obtained (in graph $H$ ) at the end of this iteration, and $h$ the number of vertices covered. Note that each $s$-tour added in a single call to minimum ratio ATSP, may use the extra $(t, s)$ arc at most once (by an averaging argument). So in case (1), the $(t, s)$ arc is absent in $\sigma$, and in case $(2)$, the $(t, s)$ arc is used exactly once \& it is the last arc in $\sigma$. Note also that during each call of minimum ratio ATSP, there is 
a feasible solution of ratio $\frac{2 O P T}{k}$ ( $\sigma^{*}$ restricted to the remaining vertices); so the ratio of the $s$-tour $\sigma, \frac{l(\sigma)}{h} \leq \rho \cdot \frac{2 O P T}{k}$. From $\sigma$ we now obtain a feasible $s$ - $t$ path $\tau$ in metric $d$ as follows. In case (1), add a direct $(s, t)$ arc: $\tau=\sigma \cdot(s, t)$; in case (2), remove the only copy of the extra $(t, s)$ arc (occurring at the end of $\sigma): \tau=\sigma \backslash\{(t, s)\}$. In either case, $s$ - $t$ path $\tau$ contains $h$ vertices and has length $d(\tau) \leq \frac{2 \rho h}{k} O P T+O P T$. Note that in case (1), $h \geq \frac{k}{2} ;$ and in case (2), since the extra $(t, s)$ arc is used, $\frac{O P T}{h} \leq \frac{l(\sigma)}{h} \leq 2 \rho \frac{O P T}{k}$, so $h \geq \frac{k}{2 \rho}$. Hence $d(\tau) \leq \frac{4 \rho h}{k} O P T$. We now greedily split $\tau$ into maximal paths, each of which has length at most $O P T$; the number of subpaths obtained is at most $\frac{d(\tau)}{O P T} \leq \frac{4 \rho h}{k}$. So one of these paths contains at least $h /\left(\frac{4 \rho h}{k}\right)=\frac{k}{4 \rho}$ vertices. Adding direct arcs from $s$ to the first vertex on this path and from the last vertex on this path to $t$, we obtain an $s-t$ path of length at most $3 \cdot O P T$ containing at least $\frac{k}{4 \rho}$ vertices

As in Blum et al. [2], we define the excess of an $s$ - $t$ di-path as the difference of the path length and the shortest path distance from $s$ to $t$. The directed min-excess problem is then: given an asymmetric metric $(V, d)$, origin $(s) \&$ destination $(t)$ vertices, and a target $k$, find an $s$ - $t$ di-path of minimum excess that visits at least $k$ other vertices. The next theorem reduces the directed minimum excess problem to the $k$-path problem, for which we just obtained an approximation algorithm.

Theorem 8 An $(\alpha, \beta)$ bi-criteria approximation algorithm for the directed $k$ path problem implies a $(2 \alpha-1, \beta)$ bi-criteria approximation algorithm for the directed minimum excess problem.

Proof: This algorithm is essentially identical to the one in Blum et al. [2] for undirected metrics; we outline the analysis here for the sake of completeness. We first show that the optimal $s$ - $t$ path $\pi$ can be divided into segments that have a certain structure. Then we show how to approximate solutions of this structure using a dynamic program.

Breaking ties arbitrarily, we assume there is an ordering $s=v_{1}, \cdots, v_{n}$ on the vertex set $V$ such that $0=d\left(s, v_{1}\right)<d\left(s, v_{2}\right)<d\left(s, v_{3}\right)<\cdots<d\left(s, v_{n}\right)$. For any real number $l \geq 0$, define $f(l)$ to be the number of $\operatorname{arcs}(x, y)$ in the optimal path $\pi$ with $d(s, x)<l \leq d(s, y)$. For values of $l \geq d(s, t)$ such that $f(l)=1$, we redefine $f(l)=2 .{ }^{1}$ Clearly $f(l) \geq 1$ for all $0 \leq l \leq d(s, t)$. We break the real line into two types of intervals: type 1 intervals are maximal intervals where $f(l)=1$, and type 2 intervals are maximal intervals where $f(l) \geq 2$. Let the interval boundaries be labeled $0=b_{1}<b_{2}<\cdots<b_{m}$; note that $b_{m-1}<d(s, t) \leq b_{m}$. Then the $i$-th interval is $\left(b_{i}, b_{i+1}\right)$, and the interval types alternate between $1 \& 2$. Let $V_{i}=\left\{v \in V: b_{i}<d(s, v) \leq b_{i+1}\right\}$ be the vertices

\footnotetext{
1 This modification is done for ease in defining the intervals as types $1 \& 2$. Without the modification, values of $l \geq d(s, t)$ with $f(l)=1$ would be placed in a type 1 interval; but the subpath of $\pi$ covering vertices in such an interval is not monotone. By ensuring that they are placed in a type 2 interval, we allow these vertices to be covered using the minimum excess algorithm. Also, the entire length of the subpath in such an interval contributes to the excess of path $\pi$; so our algorithm is allowed to approximate the length of this subpath.
} 
in the $i$-th interval; since one of any two consecutive intervals is of type 1 , the optimal path $\pi$ is monotone across vertex sets $V_{1}, \cdots, V_{m-1}$. In other words, the vertices $V_{i}$ form a contiguous subpath $S_{i}=\pi \cap V_{i}$ on $\pi$. To simplify what follows, we place vertices at interval boundaries, so every arc of $\pi$ can be assumed to be in some $V_{i}$ (as mentioned in Blum et al. [2], the analysis holds even without this assumption). So the length of $\pi$ can be written as $d(\pi)=\sum_{i} l_{i}$ where $l_{i}=d\left(S_{i}\right)$ is the length of $\pi$ in $V_{i}$. Similar to Lemma 3.1 in Blum et al. [2], we have the following.

Claim 2 If $V_{i}$ is a type 1 interval, then $l_{i} \geq b_{i+1}-b_{i}$. If $V_{i}$ is a type 2 interval not containing $t$, then $l_{i} \geq 2\left(b_{i+1}-b_{i}\right)$. If $V_{i}$ is a type 2 interval containing $t$, then $l_{i} \geq 2\left(d(s, t)-b_{i}\right)$.

If $\epsilon$ denotes the optimal value of the min-excess problem, we have $d(s, t)+\epsilon=$ $d(\pi)=\sum_{i} l_{i}$. Now from Claim $2, d(s, t)=\left(d(s, t)-b_{m-1}\right)+\sum_{i=1}^{m-2}\left(b_{i+1}-b_{i}\right) \leq$ $\sum_{i: t y p e 1} l_{i}+\frac{1}{2} \sum_{i: t y p e 2} l_{i}$. In other words, $\sum_{i: t y p e 1} l_{i}+\frac{1}{2} \sum_{i: t y p e 2} l_{i} \geq d(s, t)=$ $d(\pi)-\epsilon=\sum_{i} l_{i}-\epsilon$. Thus $\sum_{i: t y p e 2} l_{i} \leq 2 \cdot \epsilon$.

Based on this structure of the optimal solution, we write a dynamic program that stitches together intervals \& paths within them to obtain a single $s$ - $t$ di-path. The subproblems in this dynamic program are defined by tuples $(a, b, u, v, p, i)$ where $a, b \in V$ define the interval boundaries ( $a$ is closest to $s \& b$ is farthest from $s), u, v \in V$ are the start \& end vertices of the path within this interval, $p$ is the number of vertices to be covered in this interval, and $i \in\{1,2\}$ is the type of the interval.

Solving type 1 intervals: This can be done exactly in polynomial time by a simple dynamic program.

Solving type 2 intervals: This uses the directed $k$-path algorithm assumed in the theorem, to find an $\alpha$-approximately minimum length $u$ - $v$ di-path covering at least $p / \beta$ vertices.

Combining intervals: The dynamic program for combining intervals will consider the segmentation of the optimal path $\pi$ in one of its guesses. The length of the solution resulting from this is at most $\sum_{i \text { :type } 1} l_{i}+\sum_{i \text { :type } 2} \alpha \cdot l_{i}=$ $\sum_{i} l_{i}+(\alpha-1) \sum_{i: t y p e} l_{i} \leq d(\pi)+2(\alpha-1) \epsilon=d(s, t)+(2 \alpha-1) \epsilon$; so the excess of this path is at most $(2 \alpha-1) \epsilon$. Further, the number of vertices covered by any feasible solution generated by this dynamic program is at least $\frac{k}{\beta}$. Thus we get the desired bi-criteria approximation guarantee.

We now give the final reduction used in approximating directed orienteering.

Theorem 9 An $(\alpha, \beta)$ bi-criteria approximation algorithm for the directed minimum excess problem implies an $\lceil\alpha\rceil \cdot \beta$ approximation algorithm for the directed orienteering problem.

Proof: This algorithm is identical to Theorem 1 in Bansal et al. [1]. Again, we outline the proof only for the sake of completeness. Consider splitting the optimal $s$ - $t$ orienteering path $\pi$ into $l=\lceil\alpha\rceil$ pieces, each having at least $k / l$ vertices (where $k$ is the optimal value of the orienteering problem). Let the boundary vertices of these pieces be $s=u_{0}, u_{1}, \cdots, u_{l}=t$ in the order in which they appear on 
$\pi$. For vertices $u_{i} \& u_{j}$ (with $\left.i<j\right), \pi\left(u_{i}, u_{j}\right)$ denotes the subpath in $\pi$ from $u_{i}$ to $u_{j}$, and we define $\epsilon^{\prime}\left(u_{i}, u_{j}\right)=d\left(\pi\left(u_{i}, u_{j}\right)\right)-d\left(u_{i}, u_{j}\right)$ to be the excess along path $\pi$. It was proved in Bansal et al. [1] (and it also holds in the directed case) that $\epsilon^{\prime}$ is sub-additive: for any $0 \leq h<i<j \leq l, \epsilon^{\prime}\left(u_{h}, u_{i}\right)+\epsilon^{\prime}\left(u_{i}, u_{j}\right) \leq \epsilon^{\prime}\left(u_{h}, u_{j}\right)$. Among the $l$ pieces of $\pi$, consider the one $\pi\left(u_{i}, u_{i+1}\right)$ with minimum value of $\epsilon^{\prime}\left(u_{i}, u_{i+1}\right)$. Consider the $s-t$ path $\sigma=s, u_{i}, \pi\left(u_{i}, u_{i+1}\right), u_{i+1}, t$; note that $d(\pi)-d(\sigma)=\epsilon^{\prime}\left(u_{0}, u_{i}\right)+\epsilon^{\prime}\left(u_{i+1}, u_{l}\right) \geq(l-1) \cdot \epsilon^{\prime}\left(u_{i}, u_{i+1}\right)$, since the excess function $\epsilon^{\prime}$ is sub-additive and the $i$-th piece has minimum $\epsilon^{\prime}$. The algorithm guesses the optimal value $k$, vertices $u_{i}$ and $u_{i+1}$ and runs the min-excess algorithm with source $u_{i}$, destination $u_{i+1}$, and target of $k / l$ vertices. For the correct guess, $\pi\left(u_{i}, u_{i+1}\right)$ is a feasible solution, and the min-excess approximation algorithm finds a $u_{i}-u_{i+1}$ path covering at least $\frac{k}{\beta l}$ vertices, of length at most $d\left(u_{i}, u_{i+1}\right)+\alpha \cdot \epsilon^{\prime}\left(u_{i}, u_{i+1}\right)=d\left(\pi\left(u_{i}, u_{i+1}\right)\right)+(\alpha-1) \epsilon^{\prime}\left(u_{i}, u_{i+1}\right)$. So the resulting $s$ - $t$ di-path (obtained by appending $\operatorname{arcs}\left(s, u_{i}\right)$ and $\left.\left(u_{i+1}, t\right)\right)$ covers at least $\frac{k}{\beta \cdot l}$ vertices, and has length at most $d\left(s, u_{i}\right)+d\left(\pi\left(u_{i}, u_{i+1}\right)\right)+d\left(u_{i+1}, t\right)+(\alpha-$ 1) $\epsilon^{\prime}\left(u_{i}, u_{i+1}\right)=d(\sigma)+(\alpha-1) \epsilon^{\prime}\left(u_{i}, u_{i+1}\right) \leq d(\sigma)+(l-1) \epsilon^{\prime}\left(u_{i}, u_{i+1}\right) \leq d(\pi) \leq D$ the length bound.

We now obtain our main result, that relates the directed orienteering problem and minimum ratio ATSP.

Corollary 1 A $\rho$-approximation algorithm for the minimum ratio ATSP problem implies an $O(\rho)$-approximation algorithm for the directed orienteering problem. Conversely, a $\rho$-approximation algorithm for directed orienteering implies an $O(\rho)$-approximation algorithm for minimum ratio ATSP.

Proof: The first direction follows directly from Theorems 7,8 \& 9. For the other direction, we are given a $\rho$-approximation algorithm for directed orienteering. Let $D$ denote the length of some minimum ratio tour $\sigma^{*}, t$ the last vertex visited by $\sigma^{*}$ (before returning to the root $r$ ), and $h$ the number of vertices it covers; so the optimal ratio is $\frac{D}{h}$. The algorithm for minimum ratio ATSP first guesses a value $D^{\prime}$ such that $D^{\prime} \leq D \leq 2 \cdot D^{\prime}$, and the last vertex $t$. Note that we can guess powers of 2 for the value of $D^{\prime}$, which gives $O\left(\log _{2}\left(n \cdot d_{\max }\right)\right)$ possibilities for $D^{\prime}$ (where $d_{\max }$ is the length of the longest arc). Also, the number of possibilities for $t$ is at most $n$; so the algorithm only makes a polynomial number of guesses. The algorithm then runs the directed orienteering algorithm with $r \& t$ as the start/end vertices and a length bound of $2 D^{\prime}-d(t, r) \geq D-d(t, r)$. Note that removing the last $(t, r)$ arc from $\sigma^{*}$ gives a feasible solution to this orienteering instance that covers $h$ vertices. Hence the $\rho$-approximation algorithm is guaranteed to find an $r$-t di-path covering at least $\frac{h}{\rho}$ vertices, having length at most $2 D^{\prime}-d(t, r)$. Now, adding the $(t, r)$ arc to this path gives an $r$-tour of ratio at most $2 D^{\prime} /\left(\frac{h}{\rho}\right) \leq 2 \rho \frac{D}{h}$.

Corollary 1 and Theorem 5 imply an $O\left(\log ^{2} n\right)$-approximation algorithm for the directed orienteering problem. Further, any improvement in the approximation guarantee of minimum ratio ATSP implies a corresponding improvement for directed orienteering. 


\subsection{Extensions}

Discounted reward TSP: In this problem [2], we are given a metric space with rewards on vertices, and a discount factor $\gamma<1$; the goal is to find a path that maximizes the total discounted reward (where the reward for a vertex visited at time $t$ is discounted by a factor $\left.\gamma^{t}\right)$. The approximation algorithm for the undirected version of this problem (Blum et al. [2]) uses the minimum excess problem as a subroutine within a dynamic program. It can be verified directly that this reduction also works in the directed case, and so the $\left(O(1), O\left(\log ^{2} n\right)\right)$ bi-criteria approximation for directed minimum excess implies an $O\left(\log ^{2} n\right)$-approximation algorithm for directed discounted reward TSP.

Vehicle routing problem with time windows: In this VRP, we are given a metric space with a specified depot vertex and all other vertices having a time window (that specifies a release time and a deadline), and the goal is to find a path starting at the depot that maximizes the number of vertices visited in their time window. Note that orienteering is a special case when all vertices have the same time window. Bansal et al. [1] use the point-to-point orienteering problem as a subroutine, and show that an $\alpha$-approximation algorithm for orienteering implies an $O\left(\alpha \cdot \log ^{2} n\right)$-approximation for vehicle routing with time-windows. In fact, all the steps used in these reductions can be adapted to the case of directed metrics as well. So there is an $O\left(\log ^{4} n\right)$-approximation algorithm for VRP with time-windows on asymmetric metrics. A special case of the VRP with time-windows occurs when each vertex has the same release time, and only the deadline is vertex dependent; this problem is deadline TSP. The results of Bansal et al. [1] for this problem, along with the directed orienteering algorithm imply an $O\left(\log ^{3} n\right)$-approximation algorithm for directed deadline TSP.

\section{References}

1. N. Bansal, A. Blum, S. Chawla, and A. Meyerson. Approximation Algorithms for Deadline-TSP and Vehicle Routing with Time Windows. Proceedings of the 36th Annual ACM Symposium on Theory of Computing, pages 166-174, 2004.

2. A. Blum, S. Chawla, D. R. Karger, T. Lane, A. Meyerson, and M. Minkoff. Approximation Algorithms for Orienteering and Discounted-Reward TSP. Proceedings of the 44th Annual IEEE Symposium on Foundations of Computer Science, pages 46-55, 2003.

3. Chandra Chekuri, Nitish Korula, and Martin Pal. Improved Algorithms for Orienteering and Related Problems. Manuscript, 2007.

4. Chandra Chekuri and Martin Pal. A recursive greedy algorithm for walks in directed graphs. Proceedings of the 46th Annual IEEE Symposium on Foundations of Computer Science, pages 245-253, 2005.

5. M. Desrochers, J.Desrosiers, and M. Solomon. A New Optimization Algorithm for the Vehicle Routing Problem with Time Windows. Operation Research, 40:342354, 1992.

6. A. Frank. On Connectivity properties of Eulerian digraphs. Annals of Discrete Mathematics, 41, 1989. 
7. A. Frieze, G. Galbiati, and F. Maffioli. On the worst-case performance of some algorithms for the asymmetric travelling salesman problem. Networks, 12:23-39, 1982.

8. Michel X. Goemans and Dimitris J. Bertsimas. On the parsimonious property of connectivity problems. Proceedings of the 1st annual ACM-SIAM symposium on Discrete algorithms, pages 388-396, 1990.

9. B.L. Golden, L. Levy, and R. Vohra. The Orienteering Problem. Naval Research Logistics, 34:307-318, 1987.

10. M. Haimovich and A. H. G. Rinnooy Kan. Bounds and heuristics for capacitated routing problems. Mathematics of Operations Research, 10:527-542, 1985.

11. M. Held and R.M. Karp. The travelling salesman problem and minimum spanning trees. Operations Research, 18:1138-1162, 1970.

12. B. Jackson. Some remarks on arc-connectivity, vertex splitting, and orientation in digraphs. Journal of Graph Theory, 12(3):429-436, 1988.

13. M. Kantor and M. Rosenwein. The Orienteering Problem with Time Windows. Journal of the Operational Research Society, 43:629-635, 1992.

14. A. Kohen, A. R. Kan, and H. Trienekens. Vehicle Routing with Time Windows. Operations Research, 36:266-273, 1987.

15. Chung-Lun Li, David Simchi-Levi, and Martin Desrochers. On the distance constrained vehicle routing problem. Operations Research, 40:790-799, 1992.

16. W. Mader. Construction of all n-fold edge-connected digraphs (German). European Journal of Combinatorics, 3:63-67, 1982.

17. George L. Nemhauser and Laurence A. Wolsey. Integer and Combinatorial Optimization. 1999.

18. M. Savelsbergh. Local Search for Routing Problems with Time Windows. Annals of Operations Research, 4:285-305, 1985.

19. M.W.P. Savelsbergh and M. Sol. The general pickup and delivery problem. Transportation Science, 29:17-29, 1995.

20. K. C. Tan, L. H. Lee, K. Q. Zhu, and K. Ou. Heuristic Methods for Vehicle Routing Problems with Time Windows. Artificial Intelligence in Engineering, pages 281295, 2001.

21. Santosh Vempala and Mihalis Yannakakis. A convex relaxation for the asymmetric tsp. Proceedings of the 10th annual ACM-SIAM symposium on Discrete algorithms, pages $975-976,1999$.

22. David Williamson. Analysis of the held-karp heuristic for the traveling salesman problem. Master's thesis, MIT Computer Science, 1990. 